\title{
Searching for Scalar Dark Matter via Coupling to Fundamental Constants with Photonic, Atomic, and Mechanical Oscillators
}

William M. Campbell@, Ben T. McAllister, Maxim Goryachev, Eugene N. Ivanov®, and Michael E. Tobar@* ARC Centre of Excellence for Engineered Quantum Systems and ARC Centre of Excellence for Dark Matter Particle Physics, Department of Physics, University of Western Australia, 35 Stirling Highway, Crawley, Western Australia 6009, Australia

(Received 16 October 2020; revised 25 November 2020; accepted 15 January 2021; published 18 February 2021)

\begin{abstract}
We present a way to search for light scalar dark matter (DM), seeking to exploit putative coupling between dark matter scalar fields and fundamental constants, by searching for frequency modulations in direct comparisons between frequency stable oscillators. Specifically we compare a cryogenic sapphire oscillator (CSO), hydrogen maser (HM) atomic oscillator, and a bulk acoustic wave quartz oscillator (OCXO). This work includes the first calculation of the dependence of acoustic oscillators on variations of the fundamental constants, and demonstration that they can be a sensitive tool for scalar DM experiments. Results are presented based on 16 days of data in comparisons between the $\mathrm{HM}$ and OCXO, and 2 days of comparison between the OCXO and CSO. No evidence of oscillating fundamental constants consistent with a coupling to scalar dark matter is found, and instead limits on the strength of these couplings as a function of the dark matter mass are determined. We constrain the dimensionless coupling constant $d_{e}$ and combination $\left|d_{m_{e}}-d_{g}\right|$ across the mass band $4.4 \times 10^{-19} \lesssim m_{\varphi} \lesssim 6.8 \times 10^{-14} \mathrm{eV} \mathrm{c}^{-2}$, with most sensitive limits $d_{e} \gtrsim 1.59 \times 10^{-1},\left|d_{m_{e}}-d g\right| \gtrsim 6.97 \times 10^{-1}$. Notably, these limits do not rely on maximum reach analysis (MRA), instead employing the more general coefficient separation technique. This experiment paves the way for future, highly sensitive experiments based on state-of-the-art acoustic oscillators, and we show that these limits can be competitive with the best current MRA-based exclusion limits.
\end{abstract}

DOI: 10.1103/PhysRevLett.126.071301

The nature and composition of dark matter (DM) is currently one of the most pressing questions in physics. The DM particle composition and interaction properties remain unknown, despite decades of astronomical and cosmological observations, indicating that it is the dominant matter component of the Universe. Many different DM candidate particles and corresponding detection experiments have been proposed, for example [1-12], but as of yet, no confirmed detections have occurred. In this work, we investigate DM models that add ultralight scalar fields to the standard model (SM). These kinds of models consider nontrivial couplings of putative DM particles, such as the string theory dilaton, and moduli fields to the ordinary SM fields [13-17]. The introduced scalar DM particle mass is predicted to cause oscillation in some of the fundamental constants of nature, at a frequency equivalent to the DM particle mass, with measurable effects, and potentially inducing violations of Einstein's equivalence principle (EEP) [17-20]. Consequently, searching for variations in the fundamental constants, or violations of the EEP can be

Published by the American Physical Society under the terms of the Creative Commons Attribution 4.0 International license. Further distribution of this work must maintain attribution to the author(s) and the published article's title, journal citation, and DOI. Funded by SCOAP . viewed as searches for scalar dark matter candidates. Such experiments are already well developed [21-32] and are motivated from various areas of physics, beyond the search for DM.

If such DM-SM couplings exist, oscillations in fundamental constants will frequency modulate various types of clocks and oscillators, measurable via clock comparison experiments if the oscillators or clocks exhibit different dependences on the fundamental constants. Clock comparisons present a powerful tool for searching for such variations, owing to the high stability of modern frequency standards [28,33-35].

Confounding these experiments is the fact that the DM particle mass, as well as the strength of its coupling to the $\mathrm{SM}$, is unknown, and only weakly constrained [36]. As a result there is a large parameter space to search, and experiments are required that search for a range of DM particles masses, corresponding to frequency standards which are stable over different timescales.

In this work we search for variations in the fundamental constants caused by a massive scalar field constituting the local DM halo. We present limits on the possible variation of linear combinations of fundamental constants, thus placing experimental constraints on the coupling of a DM scalar field to the SM. In particular, we contribute new constraints to the relatively unexplored higher mass area of the putative scalar field's parameter space; 
$4.4 \times 10^{-19} \lesssim m_{\varphi} \lesssim 6.8 \times 10^{-14} \mathrm{eV}^{-2}$. We achieve this by monitoring frequency fluctuations of a quartz crystal bulk acoustic wave oscillator (OCXO), compared against both cryogenic sapphire oscillator (CSO) and hydrogen maser reference clocks (HM), each of which exhibits a different dependence on the fundamental constants.

We consider the model of Darmour and Donoghue [37] as implemented in Ref. [38], where $\varphi$ is a dimensionless, massive scalar field with a quadratic self-interaction potential $V(\varphi)=2\left(c^{2} / \hbar^{2}\right) m_{\varphi}^{2} \varphi^{2}$ in which the normalization has been chosen so that $m_{\varphi}$ has dimensions of mass. This model considers $\varphi$ modifications to terms in the effective action that describes the physics of ground state nuclei. At appropriately low energy scales of $\sim 1 \mathrm{GeV}$ this effective action will only contain the electron $e$, the up quark $u$ and the down quark $d$ as real particles with interactions mediated by electromagnetic $\left(A_{\mu}\right)$ and gluonic $\left(A_{\mu}^{\mathrm{A}}\right)$ fields. Weak interactions and heavy quarks are integrated out at this scale, while it is argued in Ref. [37] that EEP violation effects linked to the strange quark are relatively small, thus it is ignored here. Each of the five terms described by this effective action can then couple to $\varphi$. For this work, as is common, we consider linear couplings for these terms, thus giving a Lagrangian density for scalar field-SM interactions $\mathcal{L}_{\text {int }}$ as per Eq. (12) of Ref. [37]. Linear couplings are often considered to be the most "simple" and therefore most "compelling" couplings to the SM, taking the form

$$
\begin{aligned}
\mathcal{L}_{\text {int }}= & \varphi\left[\frac{d_{e}}{4 \mu_{0}}\left(F_{\mu \nu}\right)^{2}-\frac{d_{g} \beta_{g}}{2 g_{3}}\left(F_{\mu \nu}^{A}\right)^{2}\right. \\
& \left.-c^{2} \sum_{i=e, u, d}\left(d_{m_{i}}+\gamma_{m_{i}} d_{g}\right) m_{i} \bar{\psi}_{i} \psi_{i}\right],
\end{aligned}
$$

where $F_{\mu \nu}$ is the electromagnetic Faraday tensor, $\mu_{0}$ the magnetic permeability, $F_{\mu \nu}^{A}$ is the gluon strength tensor, $g_{3}$ the QCD gauge coupling, $\beta_{g}$ is the $\beta$ function for the running of $g_{3}, m_{i}$ is the mass of the fermions, $\gamma_{m_{i}}$ is then the anomalous dimension giving the energy running of the masses of the QCD coupled fermions, and $\psi_{i}$ denotes the fermion spinors. The constants of interest $d_{j}$ for $j=m_{u}$, $m_{d}, m_{e}, g, e$ are dimensionless coupling constants that parametrize the scalar field coupling to the SM matter fields, defining the strength of the interaction in a corresponding SM sector, and equivalently, the magnitude of any $\varphi$ dependent oscillations in the corresponding fundamental constants.

The introduction of these coupling constants into the interaction Lagrangian density will modify each term such that corresponding fundamental constants will display dependencies on $\varphi$ of the following forms:

$$
\begin{gathered}
\alpha(\varphi)=\alpha\left(1+d_{e} \varphi\right), \\
m_{i}(\varphi)=m_{i}\left(1+d_{m_{i}} \varphi\right), \quad \text { for } i=e, u, d,
\end{gathered}
$$

$$
\Lambda_{\mathrm{QCD}}(\varphi)=\Lambda_{\mathrm{QCD}}\left(1+d_{g} \varphi\right) .
$$

Here $\alpha$ is the fine structure constant, $m_{i}$ denotes fermion mass [electron $(e)$, up $(u)$ or down $(d)$ quarks], and $\Lambda_{\mathrm{QCD}}$ represents the QCD mass scale. We also note that the mean quark mass $\hat{m}=\left(m_{u}+m_{d}\right) / 2$ displays a similar dependency on $\varphi$ :

$$
\hat{m}(\varphi)=\hat{m}\left(1+d_{\hat{m}} \varphi\right) \text {, with } d_{\hat{m}}=\frac{m_{u} d_{m_{u}}+m_{d} d_{m_{d}}}{m_{u}+m_{d}} .
$$

The periodic evolution of the scalar field is given by the description in Ref. [38] which borrows from the cosmological string-theory dilaton model of Ref. [17];

$$
\varphi=\frac{4 \pi G \sigma \hbar^{2}}{m_{\varphi}^{2} c^{6}}+\varphi_{0} \cos \left(\omega_{\varphi} t+\phi\right), \text { with } \omega_{\varphi}=\frac{m_{\varphi} c^{2}}{\hbar} .
$$

Here, $\sigma=\delta \mathcal{L}_{\text {int }} / \delta \varphi$ is a source term which is due to the nonminimal coupling between the scalar field and matter. For short time periods $(t \ll 1 / H)$ this term can be considered a constant. Following the description presented in Ref. [38], we identify the scalar field as DM with energy density,

$$
\rho_{\mathrm{DM}}=\frac{c^{6}}{4 \pi G \hbar^{2}} \frac{m_{\varphi}^{2} \varphi_{0}^{2}}{2} .
$$

We see that for typical values for the local DM density of $\rho_{\text {DM }}=0.45 \mathrm{GeV} / \mathrm{cm}^{3}$ [39] the amplitude of scalar field oscillations would range from $1.84 \times 10^{-13}<\varphi_{0}<1.84 \times$ $10^{-17}$ for $1 \mathrm{mHz}<\omega_{\varphi} / 2 \pi<10 \mathrm{~Hz}$. It is thus possible to experimentally probe the coupling of a DM scalar field to matter, by searching for $\varphi$ dependent variations in dimensionless ratios of fundamental constants. This can and has been achieved by making comparison measurements between differing frequency standards whose frequency ratio is dependent on some combination of the dimensionless factors: $m_{u} / \Lambda_{\mathrm{QCD}}, m_{d} / \Lambda_{\mathrm{QCD}}, m_{e} / \Lambda_{\mathrm{QCD}}$, and $\alpha$. An experiment of this nature is thus able to probe scalar field couplings linear in $d_{e}$ and $d_{m_{i}}-d_{g}$, by considering Eqs. (2) to (7), and the local dark matter parameters.

It is often difficult to extract an exclusion limit on individual coupling parameters, owing to these combinations. There are two common methods for extracting information on individual parameters. One is known as "maximum reach analysis" (MRA) in which a series of models are considered where it is assumed that the field in question only couples to one SM sector in each model, thus excluding one non-zero coupling parameter at a time. The other method is to take multiple sets of data with differing dependencies on the constants, so that linear combinations of different parameters can be separated. This is known as "coefficient separation" and is the technique that we employ in this work. By introducing a mechanical 


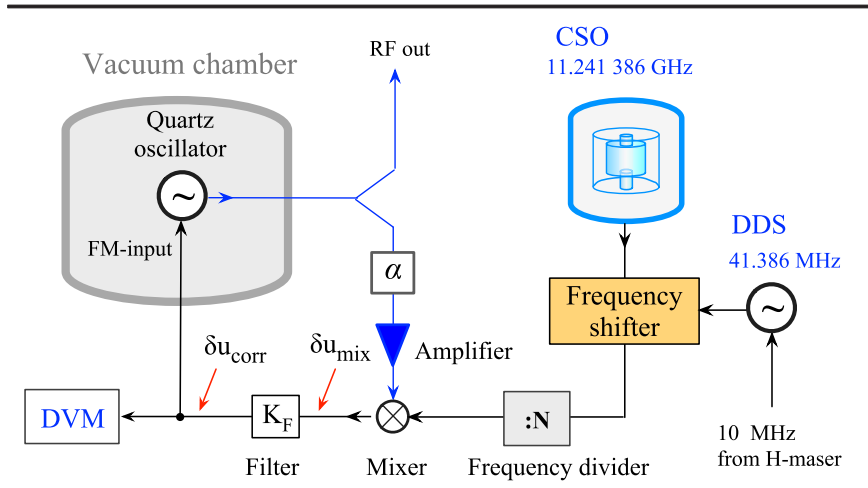

FIG. 1. Schematic diagram of the initial experimental setup of the OCXO-CSO experiment. An amplifier and attenuator $(\alpha)$ are used to prevent injection locking of the quartz oscillator. Injection locking results in spurious bright lines in the spectrum of PLL correction voltage.

resonator, which depends in a different way on the fundamental constants to various photonic and atomic oscillators, we are able to separate coefficients which have not been separated before.

In one of our experiments we analyzed fluctuations of the phase difference $\delta \phi_{21}$ between a $10 \mathrm{MHz}$ NEL Frequency Controls Inc. ultralow phase noise OCXO and a $10 \mathrm{MHz}$ signal synthesized from a microwave CSO [40]. Figure 1 shows a schematic diagram of that experiment. Here, the reference $10 \mathrm{MHz}$ signal was synthesized by shifting the CSO frequency by approximately $39 \mathrm{MHz}$ to make it sufficiently (within tens of $\mathrm{Hz}$ ) close to $11200 \mathrm{MHz}$ before dividing it 112 times. The auxiliary $39 \mathrm{MHz}$ signal was supplied by a direct digital synthesizer (DDS) phase locked to a $10 \mathrm{MHz}$ CH1-75A active HM. In another similar experiment, the $10 \mathrm{MHz}$ reference signal was supplied straight from the same HM.

A phase-locked loop (PLL) was employed to keep mean frequency of the OCXO equal to that of the $10 \mathrm{MHz}$ reference. This was necessary to permit long-term measurements of the phase difference fluctuations $\delta \phi_{21}$.

The spectral density of the $\delta \phi_{21}$ was inferred from the spectrum of PLL correction voltage $\delta u_{\text {corr }}$ (Fig. 1) via the following relationship

$S_{u}(\mathcal{F})=\left|\frac{\gamma}{1+\gamma}\right|^{2}\left(\frac{\mathcal{F}}{d f / d u}\right)^{2}\left(S_{\delta \phi}(\mathcal{F})+S_{\phi}^{\mathrm{n} / \mathrm{f}}(\mathcal{F})\right)$,

where $\mathcal{F}$ denotes the Fourier frequency, $\gamma$ is the loop gain, $d f / d u$ is the frequency-voltage tuning coefficient of the quartz oscillator, $S_{\delta \phi}$ is the spectral density of phase difference fluctuations, and $S_{\phi}^{\mathrm{n} / \mathrm{f}}$ is the spectral density of the PLL phase noise floor.

All parameters of the PLL in Eq. (8) can be determined experimentally, thus monitoring the PLL voltage signal $\delta u$ allows us to measure phase noise variations $\varphi_{21}$ that are synchronous with variations in the beat frequency $\delta f_{21}=$ $\delta f_{2}-\delta f_{1}$ of two different frequency standards.

We now consider how a variation in the fundamental constants will affect the beat frequency between each combination of these frequency standards. To do this we write the dependence of each standard's mode frequencies as a combination of the dimensionless constants $\alpha$, $m_{q} / \Lambda_{\mathrm{QCD}}$ and $m_{e} / m_{p} \propto m_{e} / \Lambda_{\mathrm{QCD}}$ [22]. Here we are assuming the constants stated above are functions of $\varphi$ as per Eqs. (2)-(4), while any other factors contained in the mode frequency dependence are true constants [41].

The derivation of the dependence of the quartz oscillator mode on the fundamental constants is discussed in the Supplemental Material [44] and is found to be given by

$$
f_{\mathrm{Q}} \propto m_{e} \alpha^{2} \sqrt{\frac{m_{e}}{m_{p}}} \propto m_{e} \alpha^{2} \sqrt{\frac{m_{e}}{\Lambda_{\mathrm{QCD}}}} .
$$

The dependencies of both the CSO and Maser frequencies are given by the relationships in Appendix A of Ref. [41], as the dependence of the CSO crystal's permittivity on $\alpha$ can be ignored at the frequencies of interest [42]. We do not consider here the sensitivity to quark mass through the spin $g$ factor of the HM transition induced by small QCD corrections [43], as its coefficient is more than an order of magnitude smaller than the other coefficients, and inclusion would cause the other coefficients to lose independence. In future work, to perform the technique of coefficient separation whilst taking into account these corrections, we would require an additional atomic oscillator with a different sensitivity to the quark mass.

$$
\begin{gathered}
f_{\mathrm{CSO}} \propto m_{e} \alpha \\
f_{\mathrm{HM}} \propto m_{e} \alpha^{4}\left(\frac{m_{e}}{m_{p}}\right) \propto m_{e} \alpha^{4}\left(\frac{m_{e}}{\Lambda_{\mathrm{QCD}}}\right) .
\end{gathered}
$$

By normalizing variations in beat frequency with respect to the shared carrier frequency $f_{0}=10 \mathrm{MHz}$, we have, for the quartz against the CSO:

$$
\begin{aligned}
\frac{\delta f_{\mathrm{CSO}}-\delta f_{\mathrm{Q}}}{f_{0}} & =-\frac{\delta \alpha}{\alpha}-\frac{1}{2}\left(\frac{\delta m_{e}}{m_{e}}-\frac{\delta \Lambda_{\mathrm{QCD}}}{\Lambda_{\mathrm{QCD}}}\right) \\
& =-\left[d_{e}+\frac{1}{2}\left(d_{m_{e}}-d_{g}\right)\right] \varphi_{0}, \\
\frac{\delta f_{\mathrm{HM}}-\delta f_{\mathrm{Q}}}{f_{0}} & =2 \frac{\delta \alpha}{\alpha}+\frac{1}{2}\left(\frac{\delta m_{e}}{m_{e}}-\frac{\delta \Lambda_{\mathrm{QCD}}}{\Lambda_{\mathrm{QCD}}}\right) \\
& =\left[2 d_{e}+\frac{1}{2}\left(d_{m_{e}}-d_{g}\right)\right] \varphi_{0},
\end{aligned}
$$

where we have used Eqs. (2)-(4), and $\varphi_{0}$ has been given by Eq. (7). 
We obtained two initial datasets as per the procedure outlined above. For the CSO-OCXO comparison, data collection took place over just 2 days, while the HMOCXO data was taken continuously over 16 days. For both datasets, PLL correction voltage time series were collected with a sampling rate of $2.2 \mathrm{~Hz}$. The spectral density of fractional frequency noise; $S_{y}$, in units of $1 / \mathrm{Hz}$, was determined by taking the Fourier transform (FT) of the beat frequency fluctuations $\delta f_{21}$ and normalizing by the carrier frequency. The range of analyzable Fourier frequencies is then determined by the sampling rate of the measurement apparatus and the total integration time. In addition to these two initial datasets, further measurements were made at later times over shorter periods in order to provide further complementary results. The CSO-OCXO and HM-OCXO experiments were sampled again, at a higher rate $(33 \mathrm{~Hz})$ for $12 \mathrm{~h}$ in order to exclude large noise sources in the initial data, as well as generating fractional frequency noise data at higher frequencies. This data will be subject to the same DM search analysis in the proceeding section, giving less stringent but complementary results. Finally, a further OCXO-OCXO control experiment which displays zero DM sensitivity was run for $12 \mathrm{~h}$, in order to characterize spurious systematic noise sources in the main data.

The goal of this analysis is to determine a limit corresponding to the weakest possible scalar field-standard model coupling strength that can be confidently excluded in the case of no detection. The general procedure is as follows. The power spectral density of fractional frequency noise, $S_{y}$, is searched for large deviations from the mean value at a range of Fourier frequencies, which would correspond to signals consistent with dark matter. A threshold "cut" value is chosen, and above this cut value any deviations from the mean are considered dark matter candidates. If all such signals can be excluded as either spurious or systematic noise, they are excluded as dark matter candidates, no detection is reported, and we move to derive exclusion limits. A signal size is determined via simulation, which corresponds to the minimum size of a dark matter signal, and thus DM-SM coupling, which we would expect to pass the chosen threshold cut value with 95\% confidence. Given we have excluded all signals above the cut, this simulation-determined signal strength corresponds to our $95 \%$ confidence exclusion limit, as we would have expected a signal of this size to remain in the data, survive the cut, and not be excluded, if it were present.

Through the utilized exclusion methods discussed in the Supplemental Material [44], we can exclude all peaks in our data as due to spurious or systematic noise. Furthermore, we are confident that should a signal containing DM characteristics arise; it would fail to be excluded by this analysis, and a strong claim of DM detection could be made.

An example of the $95 \%$ confidence exclusion limits in terms of frequency fluctuation signal strength for the

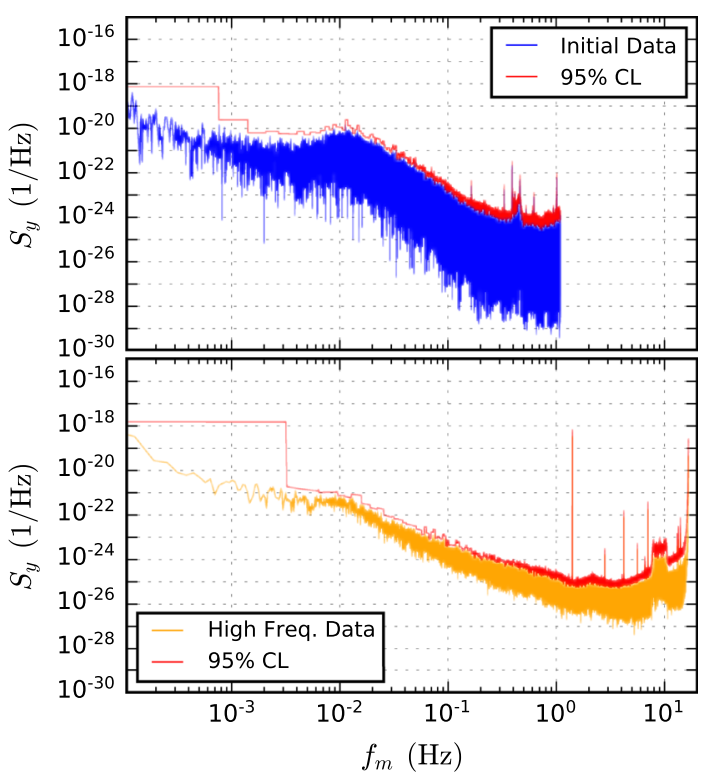

FIG. 2. Power spectral density (PSD) of frequency noise for both initial and later runs of the CSO-OCXO experiment are shown by the blue and orange traces, respectively. Also shown in red is the excluded power to $95 \%$ confidence, given by $\mathrm{MC}$ simulations. Similar confidence limits were also obtained for the HM-OCXO frequency noise data.

CSO-OCXO experiment are as shown in Fig. 2; similar limits were also computed for the HM-OCXO experiment. The excluded amplitude of frequency variation is given by the square root of these limits (when converted back from fractional to absolute frequency), which can then be substituted into Eqs. (12) to give experimental exclusion limits on two different linear combinations of $d_{e}, d_{m_{e}}$, and $d_{g}$ (a different set of coupling parameters for each type of oscillator comparison). We then utilize coefficient separation to provide further limits, effectively solving linear equations for $\left|d_{e}\right|$ and $\left|d_{m_{e}}-d_{g}\right|$. Figure 3 presents these final exclusion limits derived from both the initial sets of data, and the later higher frequency data, displayed as one combined limit.

We note that previous literature limits in this region $[25,52]$ have been approximated by applying MRA to experimental data from EEP and WEP tests. MRA is performed by effectively assuming experimental sensitivity to only one coupling parameter, setting all other parameters to zero. While this method has produced the most competitive limits to date; we note that it is an idealistic estimate, and underpinned by the inherent assumption that any cancellation between multiple parameters is unlikely $[53,54]$. In deriving our exclusion limits we have considered no case where the coupling to specific SM sectors is temporarily ignored, making them more general. Although such results are obviously inherently less sensitive, they are of slightly different and complimentary significance to those produced by MRA. 


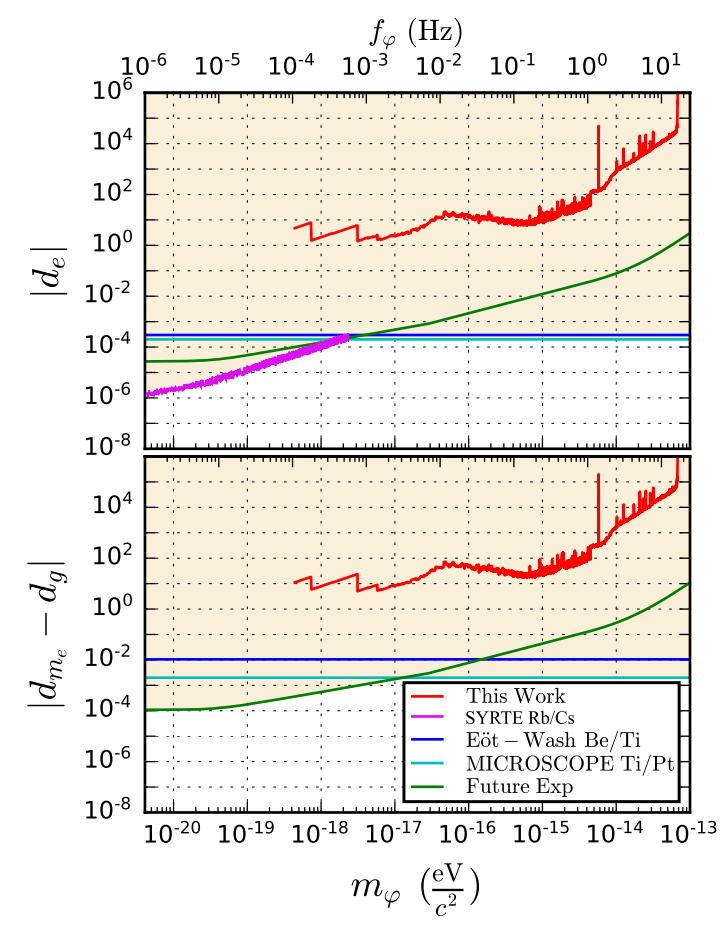

FIG. 3. Individual limits on coupling constants $\left|d_{e}\right|$ and $\left|d_{m_{e}}-d_{g}\right|$ derived in this work are presented as the red trace. This is a combined limit derived from both the initial and higher frequency data. The best known limits in the region, as approximated in Ref. [52] by performing MRA on results given by the Eöt-Wash torsion balance EP test [23] and MICROSCOPE's WEP test as well as limits on $d_{e}$ by the SYRTE fountain clock, are shown by the blue, cyan, and magenta limits, respectfully [29]. We also further present a projected estimate in green, for a potential future experiment that would utilize new low phase noise oscillators over a 5 year period.

Recent developments in CSO and OCXO oscillator technologies are giving rise to a new wave of low phase and frequency noise oscillators, far superior to the devices used in this experiment. We have included projected limits in Fig. 3, as well as frequency stability performance in Fig. 1, for a similar hypothetical experiment that searches for variations in CSO-HM and CSO-OCXO frequency differences, using current best-case noise characteristics for such devices from Refs. [28,45,46]. A 5 year experimental run time in this scenario will achieve general sensitivity limits via coefficient separation that are comparable with those produced by the approximations of MRA; however, we note that as sensitivity scales with $T^{1 / 4}$ the contribution to the improvement of these limits due to longer run times is inferior to that associated with the use of oscillators with better frequency stability. The process for determining these exclusion limits, along with the assumptions made about oscillator performance, are outlined in the Supplemental Material [44]. Further improvements to sensitivity could be made by operating the quartz oscillator in a cryogenic environment, where these oscillators see a boost in quality factor of several orders of magnitude
$[28,55,56]$. However, due to several practical challenges $[57,58]$, such a system is yet to be experimentally realized.

In conclusion, we present exclusion limits on scalar dark matter coupling to the standard model over several orders of magnitude in dark matter particle mass, based on frequency comparisons of stable oscillators. We exclude parameter space for the coupling constant $d_{e}$ and combination $d_{m_{e}}-d_{g}$. These results represent an improvable, purpose-built experimental contribution to a largely unexplored region of scalar field DM parameter space. The results we have presented are via the coefficient separation method. Although these results are several orders of magnitude less sensitive than those produced by MRA, the fact that these, and any future such limits are not reliant on the assumptions of MRA is a significant strength of this technique. The limits presented here compliment those produced by MRA, as well as other experimental searches in neighbouring regions. Additionally, these results represent the first experimental means to exclude the coupling constant combination $d_{m_{e}}-d_{g}$ in isolation, as atomic transition searches display no sensitivity to this combination. Furthermore, we present projected exclusion limits for future iterations of this experimental technique, and show that it has the potential to be competitive with the best MRA limits, without making the same assumptions. We also demonstrate for the first time the power of quartz oscillators as a tool for scalar dark matter detection, and present the derivation of the dependence of the frequencies of such resonators to the fundamental constants.

This was funded by the ARC Centre for Excellence for Engineered Quantum Systems, CE170100009, and the ARC Centre for Excellence for Dark Matter particle Physics, CE200100008, as well as ARC Grant No. DP190100071. We thank Peter Wolf for contributing useful discussions.

*michael.tobar@uwa.edu.au

[1] E. Armengaud et al., Search for low-mass WIMPs with EDELWEISS-II heat-and-ionization detectors, Phys. Rev. D 86, 051701 (2012).

[2] C. E. Aalseth, P. S. Barbeau, J. Colaresi, J. I. Collar, J. D. Leon, J. E. Fast, N. E. Fields, T. W. Hossbach, A. Knecht, M. S. Kos, M. G. Marino, H. S. Miley, M. L. Miller, J. L. Orrell, and K. M. Yocum (CoGeNT Collaboration), A search for low-mass dark matter using $p$-type point contact germanium detectors, Phys. Rev. D 88, 012002 (2013).

[3] R. Essig et al., Dark sectors and new, light, weakly-coupled particles, arXiv:1311.0029.

[4] G. Angloher et al., Results on light dark matter particles with a low-threshold CRESST-II detector, Eur. Phys. J. C 76, 25 (2016).

[5] J. M. Gaskins, A review of indirect searches for particle dark matter, Contemp. Phys. 57, 496 (2016). 
[6] N. Du et al. (ADMX Collaboration), Search for Invisible Axion Dark Matter with the Axion Dark Matter Experiment, Phys. Rev. Lett. 120, 151301 (2018).

[7] L. Zhong et al., Results from phase 1 of the HAYSTAC microwave cavity axion experiment, Phys. Rev. D 97, 092001 (2018).

[8] P. Sikivie, Invisible axion search methods, arXiv:2003.02206.

[9] S. Gori, G. Perez, and K. Tobioka, KOTO vs. NA62 dark scalar searches, arXiv:2005.05170.

[10] L. Badurina et al. (AION Collaboration), An atom interferometer observatory and network, J. Cosmol. Astropart. Phys. 05 (2020) 011.

[11] B. T. McAllister, G. Flower, E. N. Ivanov, M. Goryachev, J. Bourhill, and M. E. Tobar, The organ experiment: An axion haloscope above $15 \mathrm{GHz}$, Phys. Dark Universe 18, 67 (2017).

[12] A. Caldwell, G. Dvali, B. Majorovits, A. Millar, G. Raffelt, J. Redondo, O. Reimann, F. Simon, and F. Steffen (MADMAX Working Group), Dielectric Haloscopes: A New Way to Detect Axion Dark Matter, Phys. Rev. Lett. 118, 091801 (2017).

[13] M. B. Green, J. H. Schwarz, and E. Witten, SuperstringTheory (Cambridge University Press, Cambridge, England, 1988).

[14] M. Gasperini, F. Piazza, and G. Veneziano, Quintessence as a runaway dilaton, Phys. Rev. D 65, 023508 (2001).

[15] T. Damour, F. Piazza, and G. Veneziano, Runaway Dilaton and Equivalence Principle Violations, Phys. Rev. Lett. 89, 081601 (2002).

[16] T. Damour and J.F. Donoghue, Phenomenology of the equivalence principle with light scalars, Classical Quantum Gravity 27, 202001 (2010).

[17] T. Damour and A. Polyakov, The string dilation and a least coupling principle, Nucl. Phys. B423, 532 (1994).

[18] T. Damour, G. W. Gibbons, and C. Gundlach, Dark Matter, Time-Varying G, and a Dilaton Field, Phys. Rev. Lett. 64, 123 (1990).

[19] Y. Stadnik and V. Flambaum, Can Dark Matter Induce Cosmological Evolution of the Fundamental Constants of Nature?, Phys. Rev. Lett. 115, 201301 (2015).

[20] Y. V. Stadnik and V. V. Flambaum, Searching for Dark Matter and Variation of Fundamental Constants with Laser and Maser Interferometry, Phys. Rev. Lett. 114, 161301 (2015).

[21] M. E. Tobar, P. L. Stanwix, J. J. McFerran, J. Guena, M. Abgrall, S. Bize, A. Clairon, P. Laurent, P. Rosenbusch, D. Rovera, and G. Santarelli, Testing local position and fundamental constant invariance due to periodic gravitational and boost using long-term comparison of the SYRTE atomic fountains and H-masers, Phys. Rev. D 87, 122004 (2013).

[22] M. E. Tobar, P. Wolf, S. Bize, G. Santarelli, and V. Flambaum, Testing local lorentz and position invariance and variation of fundamental constants by searching the derivative of the comparison frequency between a cryogenic sapphire oscillator and hydrogen maser, Phys. Rev. D 81, 022003 (2010).

[23] S. Schlamminger, K.-Y. Choi, T. A. Wagner, J. H. Gundlach, and E. G. Adelberger, Test of the Equivalence Principle
Using a Rotating Torsion Balance, Phys. Rev. Lett. 100, 041101 (2008).

[24] G. L. Smith, C. D. Hoyle, J. H. Gundlach, E. G. Adelberger, B. R. Heckel, and H.E. Swanson, Short-range tests of the equivalence principle, Phys. Rev. D 61, 022001 (1999).

[25] A. Arvanitaki, J. Huang, and K. V. Tilburg, Searching for dilaton dark matter with atomic clocks, Phys. Rev. D 91, 015015 (2015)

[26] K. Van Tilburg, N. Leefer, L. Bougas, and D. Budker, Search for Ultralight Scalar Dark Matter with Atomic Spectroscopy, Phys. Rev. Lett. 115, 011802 (2015).

[27] J. Manley, D. J. Wilson, R. Stump, D. Grin, and S. Singh, Searching for Scalar Dark Matter with Compact Mechanical Resonators, Phys. Rev. Lett. 124, 151301 (2020).

[28] M. Goryachev, Z. Kuang, E. N. Ivanov, P. Haslinger, H. Muller, and M. E. Tobar, Next generation of phonon tests of lorentz invariance using quartz BAW resonators, IEEE Trans. Ultrason. Ferroelectr. Freq. Control 65, 991 (2018).

[29] J. Bergé, P. Brax, G. Métris, M. Pernot-Borràs, P. Touboul, and J.-P. Uzan, MICROSCOPE Mission: First Constraints on the Violation of the Weak Equivalence Principle by a Light Scalar Dilaton, Phys. Rev. Lett. 120, 141101 (2018).

[30] E. Savalle, A. Hees, F. Frank, E. Cantin, P.-E. Pottie, B. M. Roberts, L. Cros, B. T. McAllister, and P. Wolf, Searching for Dark Matter with an Unequal Delay Interferometer: The Damned Experiment, arXiv:2006.07055 [Phys. Rev. Lett. (to be published)].

[31] Y. V. Stadnik and V. V. Flambaum, Enhanced effects of variation of the fundamental constants in laser interferometers and application to dark-matter detection, Phys. Rev. A 93, 063630 (2016).

[32] H. Grote and Y. V. Stadnik, Novel signatures of dark matter in laser-interferometric gravitational-wave detectors, Phys. Rev. Research 1, 033187 (2019).

[33] S. Galliou, M. Goryachev, R. Bourquin, P. Abbé, J. P. Aubry, and M.E. Tobar, Extremely low loss phonontrapping cryogenic acoustic cavities for future physical experiments, Sci. Rep. 3, 2132 (2013).

[34] M. Abgrall, J. Guéna, M. Lours, G. Santarelli, M. E. Tobar, S. Bize, S. Grop, B. Dubois, C. Fluhr, and V. Giordano, High-stability comparison of atomic fountains using two different cryogenic oscillators, IEEE Trans. Ultrason. Ferroelectr. Freq. Control 63, 1198 (2016).

[35] M. Goryachev, E. N. Ivanov, M. E. Tobar, and S. Galliou, Bulk acoustic wave resonator-oscillators and tests of fundamental physics, in 2019 Joint Conference of the IEEE International Frequency Control Symposium and European Frequency and Time Forum (EFTF/IFC) (2019), pp. 1-2.

[36] J. R. Primack, D. Seckel, and B. Sadoulet, Detection of cosmic dark matter, Annu. Rev. Nucl. Part. Sci. 38, 751 (1988).

[37] T. Damour and J.F. Donoghue, Equivalence principle violations and couplings of a light dilaton, Phys. Rev. D 82, 084033 (2010).

[38] A. Hees, J. Guéna, M. Abgrall, S. Bize, and P. Wolf, Searching for an Oscillating Massive Scalar Field as a Dark Matter Candidate Using Atomic Hyperfine Frequency Comparisons, Phys. Rev. Lett. 117, 061301 (2016). 
[39] P. J. McMillan, Mass models of the milky way, Mon. Not. R. Astron. Soc. 414, 2446 (2011).

[40] J. G. Hartnett, N. R. Nand, and C. Lu, Ultra-low-phasenoise cryocooled microwave dielectric-sapphire-resonator oscillators, Appl. Phys. Lett. 100, 183501 (2012).

[41] J. P. Turneaure, C. M. Will, B. F. Farrell, E. M. Mattison, and R. F. C. Vessot, Test of the principle of equivalence by a null gravitational red-shift experiment, Phys. Rev. D 27, 1705 (1983).

[42] M. E. Tobar and J. G. Hartnett, Proposal for a new test of the time independence of the fine structure constant $\alpha$ using orthogonally polarized whispering gallery modes in a single sapphire resonator, Phys. Rev. D 67, 062001 (2003).

[43] V. V. Flambaum, D. B. Leinweber, A. W. Thomas, and R. D. Young, Limits on variations of the quark masses, QCD scale, and fine structure constant, Phys. Rev. D 69, 115006 (2004).

[44] See Supplemental Material at http://link.aps.org/ supplemental/10.1103/PhysRevLett.126.071301 for derivations for the dependence of the oscillators used on the fundamental constants, as well as further discussions on the analysis methods presented in the main text, which includes Refs. [28,39,45-51].

[45] E. N. Ivanov and M. E. Tobar, Generation of spectrally pure microwave signals, arXiv:2003.09117.

[46] MHM-2020 Active Hydrogen Maser, Microchip Technology Inc (2020).

[47] L. c. v. F. Pašteka, Y. Hao, A. Borschevsky, V. V. Flambaum, and P. Schwerdtfeger, Material Size Dependence on Fundamental Constants, Phys. Rev. Lett. 122, 160801 (2019).

[48] D. J. Griffiths, Introduction to Quantum Mechanics (Prencitce Hall, Englewood Cliffs, NJ, 1995).
[49] J. Gilman, Micromechancis of Flow in Solids (McGraw-Hill, New York, NY, 1969).

[50] E. J. Daw, A search for halo axions, Ph.D. Thesis, Massachusetts Institute of Technology, 1998.

[51] G. P. Centers, J. W. Blanchard, J. Conrad, N. L. Figueroa, A. Garcon, A. V. Gramolin, D. F. J. Kimball, M. Lawson, B. Pelssers, J. A. Smiga, A. O. Sushkov, A. Wickenbrock, D. Budker, and A. Derevianko, Stochastic fluctuations of bosonic dark matter, arXiv:1905.13650.

[52] A. Hees, O. Minazzoli, E. Savalle, Y. V. Stadnik, and P. Wolf, Violation of the equivalence principle from light scalar dark matter, Phys. Rev. D 98, 064051 (2018).

[53] A. Bourgoin, C. L. Poncin-Lafitte, A. Hees, S. Bouquillon, G. Francou, and M.-C. Angonin, Lorentz Symmetry Violations from Matter-Gravity Couplings with Lunar Laser Ranging, Phys. Rev. Lett. 119, 201102 (2017).

[54] N. A. Flowers, C. Goodge, and J. D. Tasson, SuperconductingGravimeter Tests of Local Lorentz Invariance, Phys. Rev. Lett. 119, 201101 (2017).

[55] A. Lo, P. Haslinger, E. Mizrachi, L. Anderegg, H. Müller, M. Hohensee, M. Goryachev, and M. E. Tobar, Acoustic Tests of Lorentz Symmetry Using Quartz Oscillators, Phys. Rev. X 6, 011018 (2016).

[56] E. Ivanov, M. Goryachev, M. Tobar, and S. Galliou, Pound stabilized cryogenic bulk acoustic wave resonator-oscillator, in 2018 IEEE International Frequency Control Symposium (IFCS 2018) (IEEE, Olympic Valley, 2013).

[57] M. Goryachev, S. Galliou, P. Abbe, P.-Y. Bourgeois, S. Grop, and B. Dubois, Quartz resonator instabilities under cryogenic conditions, IEEE Trans. Ultrason. Ferroelectr. Freq. Control 59, 21 (2012).

[58] M. Goryachev, S. Galliou, J. Imbaud, and P. Abbé, Advances in development of quartz crystal oscillators at liquid helium temperatures, Cryogenics 57, 104 (2013). 